\title{
Hoarseness Due to Right Vocal Cord Paralysis Associated with Aortic Diverticulum from Right Aortic Arch- A Rare and Unusual Vascular Etiology of Right Vocal Cord Paralysis
}

\author{
Produl Hazarika1* ${ }^{*}$, Seema E. Punnoose ${ }^{1}$, Sanjay Arora1, \\ Ramagowdanapura Sadashivan Diesh ${ }^{2}$, Raghavendra K. Itgampalli3 ${ }^{3}$, Rohit Singh4 \\ ${ }^{1}$ Department of ENT, NMC Specialty Hospital, Abu Dhabi, UAE \\ ${ }^{2}$ Department of Cardiothoracic Surgery, NMC Specialty Hospital, Abu Dhabi, UAE \\ ${ }^{3}$ Department of Radiology, NMC Specialty Hospital, Abu Dhabi, UAE \\ ${ }^{4}$ Department of ENT \& Head-Neck Surgery, Kasturba Medical College, Manipal, India \\ Email: produl_ent@rediffmail.com, seema1p@yahoo.com, sanjayaroraent@gmail.com, \\ donusada@yahoo.co.in, raghavitgampalli@gmail.com, rohit.singh@gmail.com
}

Received 14 February 2015; accepted 3 March 2015; published 10 March 2015

Copyright (C) 2015 by authors and Scientific Research Publishing Inc.

This work is licensed under the Creative Commons Attribution International License (CC BY).

http://creativecommons.org/licenses/by/4.0/

(c) (i) Open Access

\begin{abstract}
Right vocal cord paralysis in our present case was diagnosed on clinical and radiological examination which is precipitated by an anomalous right aortic arch with diverticulum. This is a very uncommon vascular etiology of hoarseness and is extremely rare. Because of this rarity, the practicing otolaryngologist may miss this finding while evaluating a case of idiopathic right vocal cord paralysis. Thus, the authors feel that idiopathic or unexplained right vocal cord paralysis should be routinely investigated with a CT or MRI of neck and chest with or without contrast to avoid such shortcomings. There is only one such case of right vocal cord paralysis by right aortic which has been reported earlier in literature.
\end{abstract}

\section{Keywords}

Hoarseness, Vocal Cord Paralysis, Right Aortic Arch, CT Scan Neck and Chest

\footnotetext{
${ }^{*}$ Corresponding author.
}

How to cite this paper: Hazarika, P., et al. (2015) Hoarseness Due to Right Vocal Cord Paralysis Associated with Aortic Diverticulum from Right Aortic Arch-A Rare and Unusual Vascular Etiology of Right Vocal Cord Paralysis. International Journal of Otolaryngology and Head \& Neck Surgery, 4, 99-103. http://dx.doi.org/10.4236/ijohns.2015.42018 


\section{Introduction}

Hoarseness due to unilateral or bilateral vocal cord paralysis is the common presenting symptom in laryngological practice. In fact, hoarseness is the first presenting symptom of various laryngeal diseases and fortunately most of the primary diseases of the larynx can be evaluated either by rigid or flexible laryngoscopy with or without biopsy and histopathology. However, neurogenic vocal cord paralysis causing hoarseness necessitates detailed evaluation as the cause of paralysis may be distal or proximal to the larynx. We herein present one such case of hoarseness with right vocal cord paralysis caused by very rarely seen right aortic arch with no cardiac or thoracic symptoms or complaints except mild essential hypertension. He did not have any visits to the cardiothoracic unit prior to our referral. Google and Pubmed search showed only one such case that had been reported earlier in KBB-Forum (2013) — A Turkish National Journal of Otolaryngology. This paper highlights the rarity of this condition and the need to include CT or MRI of neck and chest to evaluate idiopathic right vocal cord paralysis.

\section{Case Report}

Mr. A.R.P.A., 65 years old Indian male patient attended the ENT clinic of NMC Specialty Hospital, Abu Dhabi on $17^{\text {th }}$ May 2011 for his complaints of nasal blocking, cough, fever, throat pain of one week duration associated with hoarseness since 7 years. Hoarseness had become progressively worse since last one week. He stated that he had undergone microlaryngoscopy and biopsy twice in the past on $15^{\text {th }} \& 31^{\text {st }}$ May 2007 to rule out laryngeal pathology for hoarseness. He is a known hypertensive on antihypertensive medication since the last 5 years. Both his biopsy reports were negative for any specific pathology. He was diagnosed and treated for Acute Upper Respiratory Tract Infection in our clinic for symptoms of cough and change in voice with a follow up advice after 15 days for evaluation of hoarseness. He did not show up for follow up as advised. His next visit to our clinic was almost after three years on $23^{\text {rd }}$ May 2014 and a video laryngoscopy revealed right vocal cord in the right paramedian position with restricted mobility and a phonatory gap. Right arytenoid was seen falling forward with no obvious growth to be seen in the larynx. CT scan of neck and upper chest with contrast was done on $5^{\text {th }}$ June 2014. CT scan showed enlarged inferior recess of right pyriform sinus, medially displaced right aryepiglottic fold, anteromedial displacement of right arytenoid cartilage with features of right vocal cord paralysis (Figure 1). Visualized sections of thorax revealed right aortic arch with mirror image branching with small suspicious Kommerrell's diverticulum with a pressure effect over oesophagus and at tracheo-esophageal groove. A cardiothoracic consultation was sought due to the rarity of the aortic arch anomaly on $13^{\text {th }}$ June 2014 . A repeat CT scan with contrast of the whole chest was advised by the cardiothoracic surgeon on $28^{\text {th }}$ June 2014 . It revealed a right aortic arch with mirror image branching with aortic diverticulum measuring approximately $15 \mathrm{~mm} \times 14$ mm along medial border causing pressure effect over oesophagus at D2-D3 level and at tracheoesophageal groove (Figure 2). Figure 3 shows volume rendered image of right aortic arch showing mirror image branching and Figure 4 shows volume reduced image of thoracic aorta showing aortic diverticulum from medial wall of

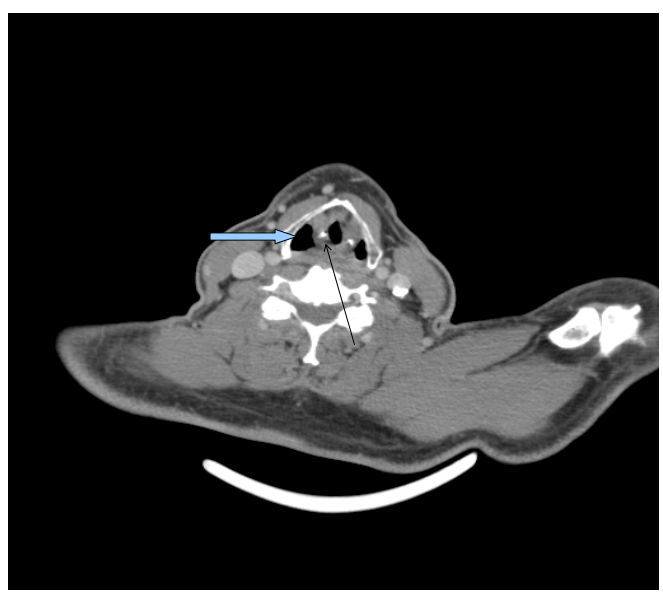

Figure 1. Axial CT contrast at the level of thyroid cartilage shows enlarged inferior recess of right pyriform fossa (block arrow) and anteromedial displacement of right arytenoids cartilage (thin arrow). 


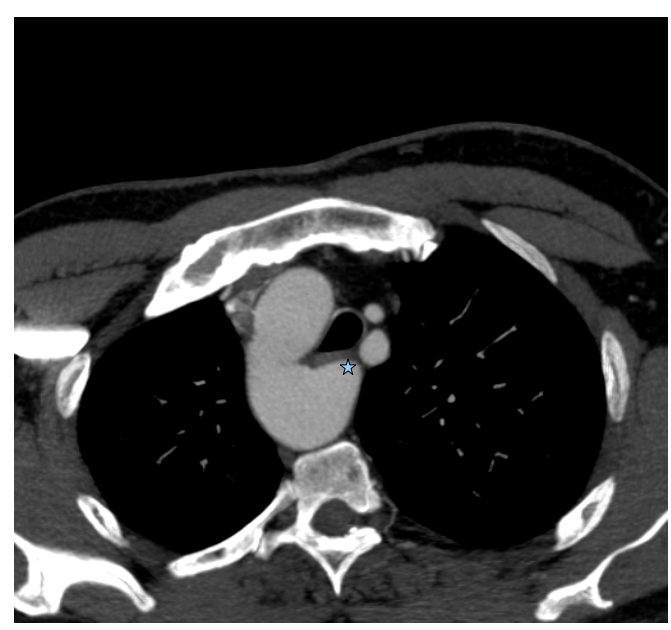

Figure 2. Axial contrast CT at the level of aortic arch shows Right sided aortic arch with an aortic diverticulum (asterix) along medial wall causing pressure effect over oesophagus/tracheosophageal groove.

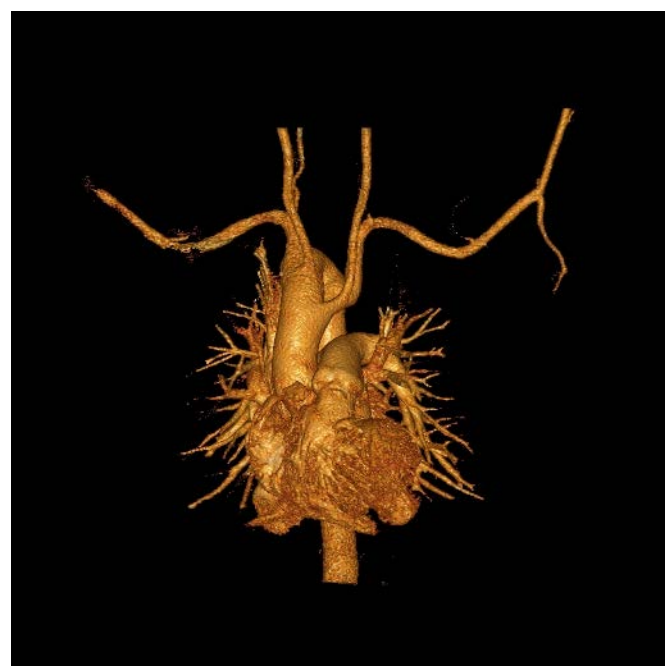

Figure 3. Volume rendered image of right aortic arch showing mirror image branching.

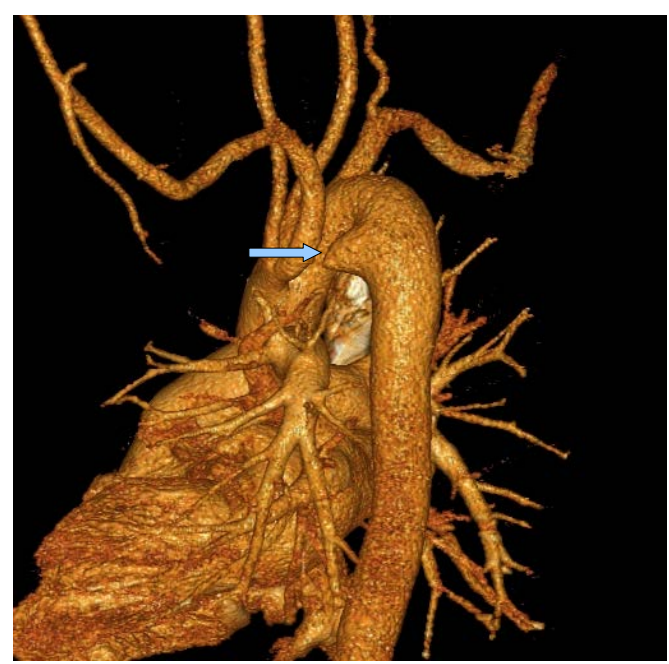

Figure 4. Volume reduced image of thoracic aorta showing aortic diverticulum (block arrow) from medial wall of aorta. 
aorta. All these findings were discussed with the patient and our surgical colleague and a wait and watch policy was decided upon for the treatment of the diverticulum. For the symptom of hoarseness phonosurgical procedure was advised. An informed consent was also taken from the patient for the presentation.

\section{Discussion}

Neurogenic vocal cord paralysis may be unilateral or bilateral and can be caused by dysfunction of either the recurrent laryngeal nerve or the main vagus nerve trunk. Recurrent laryngeal nerve paralysis can be caused by various pathologies like inflammation, trauma, neurogenic or neuromuscular disease, neoplastic, viral, iatrogenic, idiopathic, vascular and cardiac diseases [1]. The recurrent laryngeal nerve paralysis associated with cardiovascular pathology is termed as cardiovocal syndrome or ortners syndrome [2]-[4]. The pathology of the cardiovocal syndrome generally includes aneurysm or cardiac dilatation causing compression injury mostly in left recurrent laryngeal nerve in the thorax. Left recurrent nerve palsy of cardiogenic origin has been well documented in the literature [5]. This is because left recurrent laryngeal nerve has a close relation to the left aortic arch. However, right recurrent laryngeal nerve injury causing right vocal cord paralysis due to right aortic arch without any other cardiac anomaly is extremely rare and so far only one such case has been reported in the literature [3]. This could have happened because of the abnormal origin of right recurrent laryngeal nerve from right vagus in thorax [6].

Anatomically, the left recurrent laryngeal nerve arises from left vagus at the level of left aortic arch, then hooks back posteriorly under the arch and ascends through superior mediastinum to reach the groove between the trachea and oesophagus. Left recurrent laryngeal nerve is more prone for paralysis than the right side because it pursues a longer intrathoracic course, coming into contact with the mediastinal surface of left lung, continuing along the mediastinal lymph nodes and finally looping around the aortic arch. Therefore, cardiac dilatation, aneurysm etc. can easily precipitate left vocal cord paralysis. However, right recurrent laryngeal nerve almost always springs off in the root of the neck and there is absolutely no chance of getting it affected in cardiac dilatation or aneurysm unless it has an abnormal origin from the right vagus in the thorax. Anatomical variations in the course of right recurrent laryngeal nerve are very common. But right recurrent nerve taking origin in the thorax instead of root of the neck is a very rare anomaly. Of the variation, the occurrence of non-recurrent laryngeal nerve is more common on the right side $(0.6 \%)$ than on the left side $(0.04 \%)$ [6] [7]. Variations are associated with the vascular anomalies such as an aberrant origin of the right subclavian artery from descending thoracic aorta or right sided aortic arch. When there is avascular anomaly of the right subclavian artery, right recurrent laryngeal nerve no longer recurs around the artery but proceeds from the vagus nerve in a more transverse direction to the larynx [4].

Right aortic arch is generally an asymptomatic congenital anomaly seen in $0.1 \%$ of the population [8]. Felson and Palayew (1963) mentioned mainly of two types of right aortic arch with their different branching patterns [9]. First one is the origin of brachiocephalic vessels from the arch in a mirror image fashion where the first branch is left innominate artery followed by right common carotid artery and then the right subclavian artery. The second type will have left common carotid artery as the first branch followed by right common carotid, right subclavian and then an aberrant left subclavian artery from the descending aorta via a diverticulum [10]. Right carotid arch with mirror image branching is almost always associated with congenital cardiac anomalies like Fallot's tetralogy with or without pulmonary atresia or truncusarteriosus. It is extremely rare to find a case like ours where there is right aortic arch with mirror image branching with no congenital cardiac anomalies but having right vocal cord paralysis. CT finding in the present case showed aortic diverticulum along the medial border causing pressure effect over oesophagus at D2-D3 level and tracheoesophageal groove. It is reported in the literature that right recurrent laryngeal nerve very rarely takes origin in the thorax in association with vascular anomalies like aberrant origin of right subclavian artery from descending thoracic aorta or right subclavian artery.

Right vocal cord palsy in our present case may be due to the compression injury of the nerve in the tracheoesophageal groove by the aortic diverticulum but not by any inflammation or any growth infiltration. Therefore, repeated microlaryngoscopy and biopsy failed to show any local pathology in larynx. This form of right vocal cord palsy is extremely rare and only one case has been reported previously. The possibility of this pathology has naturally been missed in previous laryngological examinations due to such an uncommon etiology of the vocal cord paralysis. So, the authors feel that an unexplained right vocal cord palsy should be further evaluated 
by performing a CT or MRI of neck and chest. This type of vocal cord paralysis perhaps should be considered as an ideal indication for phonosurgery, if acceptable to the patient after the management of his primary cause of hoarseness. A successful phonosurgical procedure will improve the voice quality and thus carries good prognosis. The cardiothoracic team of experts have recommended endovascular stenting of aorta to prevent an increase in size of diverticulum and rupture.

\section{Acknowledgements}

We, the authors acknowledge the immense help and support that we received from our NMC group medical director Dr. B. R. Shetty and Medical Director of NMC Specialty Hospital, Abu Dhabi Dr. C. R. Shetty. Also we are grateful to the Department of ENT \& Head-Neck Surgery of Kasturba Medical College of Manipal University, India for helping us in preparation of this manuscript.

\section{References}

[1] Titche, L.L. (1976) Causes of Recurrent Laryngeal Nerve Paralysis. Archives of Otolaryngology, 102, $259-261$. http://dx.doi.org/10.1001/archotol.1976.00780100045002

[2] Omer, K., Hakan, B., Umit, A., Ozeyir, Y., Turan, I. and Burak, A. (2013) Recurrent Vocal Cord Paralysis Associated with Right Aortic Arch. KBB-Forum, 12.

[3] Annema, J.T., Brahim, J.J. and Rabe, K.F. (2004) A Rare Case of Ortners Syndrome (Cardiovocal Hoarseness). Thorax, 59, 636-637. http://dx.doi.org/10.1136/thx.2003.020503

[4] Zaki, S.A., Asif, S. and Shanbag, P. (2010) Ortner Syndrome in Infants. Indian Pediatrics, 47, 351-353. http://dx.doi.org/10.1007/s13312-010-0053-y

[5] Song, S.W., Jun, B.C., Cho, K.J., Lee, S., Kim, Y.J. and Park, S.H. (2011) CT Evaluation of Vocal Cord Paralysis Due to Thoracic Diseases: A 10 Years Retrospective Study. Yonsei Medical Journal, 52, 831-837. http://dx.doi.org/10.3349/ymj.2011.52.5.831

[6] Moosman, D.A. and DeWeese, M.S. (1968) The External Laryngeal Nerve as Related to Thyroidectomy. Surgery, Gynecology Obstetrics, 127, 1101.

[7] Aniruddha, S. (2012) A Case Report of Abnormal Origin of Right Recurrent Laryngeal Nerve from Right Vagus in Thorax. IJCRI, 3, 4-7. http://dx.doi.org/10.5348/ijcri-2012-03-97-CR-2

[8] Ballotta, E., Mion, E. and Bardini, R. (2003) Right Sided Aortic Arch and Aberrant Left Subclavianartery. Journal of Cardiovascular Surgery (Torino), 44, 783-784.

[9] Felson, W. and Palayew, M.J. (1963) Two Types of Right Aortic Arch. Radiology, 81, 745-759. http://dx.doi.org/10.1148/81.5.745

[10] McElhinney, D.B., Hoydu, A.K., Gaynor, J.W., Spray, T.L., Goldmuntz, E. and Weinberg, P.M. (2001) Patterns of Right Aortic Arch and Mirror-Image Branching of the Brachiocephalic Vessels without Associated Anomalies. Pediatric Cardiology, 22, 285-291. http://dx.doi.org/10.1007/s002460010231 